\title{
Pancreatitis, panniculitis and polyarthritis
}

\author{
Steven J. Katz ${ }^{1}$, Alison Kydd ${ }^{2}$, Elaine A. Yacyshyn ${ }^{1}$ \\ 1. Division of Rheumatology, University of Alberta, Edmonton, Alberta, Canada. 2. Division of Rheumatology, University of \\ British Columbia, Nanaimo, British Columbia, Canada \\ Correspondence: Steven J. Katz, MD, FRCPC. Address: 562 Heritage Medical Research Centre, University of Alberta, \\ Edmonton, AB, Canada. E-mail: steven.katz@ualberta.ca
}

Received: December 22, 2013 Accepted: January 14, $2014 \quad$ Online Published: January 16, 2014

DOI : $10.5430 /$ crim.v1n1p1

URL: http://dx.doi.org/10.5430/crim.v1n1p1

\section{Abstract}

Objectives: To present a case of pancreatitis, panniculitis and polyarthritis syndome, including the full spectrum of the arthritis component.

Methods: A case of PPP syndrome was examined, with a detailed review of the patient's synovial fluid aspirate including cell count, crystals, culture and a lipid analysis which was compared to a control patient.

Results: The synovial fluid aspirate from PPP syndrome demonstrates unique crystal formation and higher lipids.

Conclusion: PPP syndrome is a rare condition which can be difficult to diagnose. A lipid analysis of a patient's synovial fluid aspirate may be a useful tool to aid in the diagnosis.

\section{Key words}

Pancreatitis, Panniculitis, Polyarthritis, Rheumatology

\section{I ntroduction}

Polyarthritis presenting in a triad with pancreatitis and panniculitis is an uncommon clinical presentation. So called PPP syndrome, it is rarely reported. The polyarthritis can be easily misdiagnosed not only due to the condition's rarity, but the remaining manifestations can be easily missed. However, appropriate diagnosis is important both to estimate prognosis and to prevent inappropriate use of treatment modalities. While PPP syndrome has been previously described, the entire spectrum of the arthritic component has not. We describe a case of PPP syndrome, demonstrate the results of synovial aspirate including a lipid analysis, and rapid plain film imaging changes.

\section{Case presentation}

\subsection{History}

Mr. S was a 72 year old Caucasian male with a past history of osteoarthritis, depression, possible chronic alcohol dependence, and bilateral inguinal hernia repair remotely. He took no regular medications. He was in good health until 10 days prior to presentation when he first noticed pain and swelling of his hands, worse on the right than the left side. These symptoms rapidly worsened and 2-days prior to his presentation he developed pain and swelling to his ankles bilaterally 
such that he was unable to ambulate. He had also developed fluctuant raised skin lesions overlying his involved joints. The patient denied constitutional symptoms but did report a mild self-limited diarrheal illness one month prior to the onset of symptoms. He had not experienced any other respiratory, gastrointestinal or genitourinary symptoms and had no prior history of joint effusions or skin conditions. Due to concern for alcohol withdrawal at a peripheral hospital, the patient was started on chlordiazepoxide and lorazepam as needed. He had also been started on prednisone 50mg daily for his joint pain, but with minimal relief.

\subsection{Physical examination}

Pertinent findings on physical examination revealed normal vital signs with head and neck, neurologic, respiratory and cardiac examinations all normal. Abdominal exam revealed no tenderness, nor hepatosplenomegaly. Musculoskeletal exam revealed synovitis and diffuse swelling in most small joints of the hands, knees, ankles and small joints of the feet. The patient had numerous raised 1-4 centimeter in diameter erythematous fluctuant lesions over the distal extremities (see Figure 1).

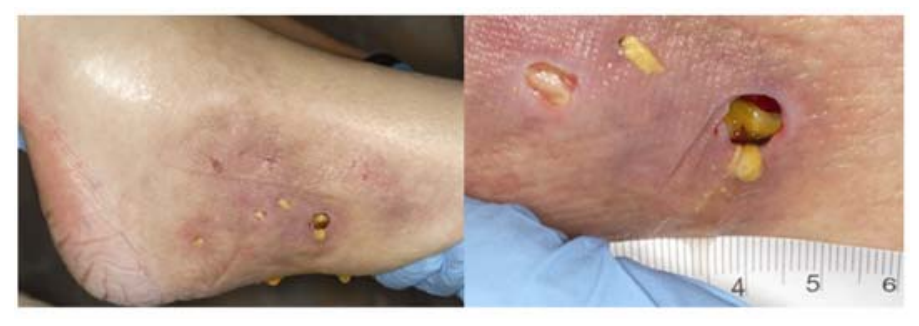

Figure 1. Fluctuant lipid lesions by patient's ankle

\subsection{I nvestigations}

Investigations showed hemoglobin of $109 \mathrm{~g} / \mathrm{L}$, a leukocytosis of $54.2 \times 10^{9} / \mathrm{L}$, alkaline phosphatase $154 \mathrm{IU} / \mathrm{L}$, lactate dehydrogenase 397 IU/L, ESR $74 \mathrm{~mm} / \mathrm{h}$ and CRP $262 \mathrm{mg} / \mathrm{L}$. All immunologic studies were negative except for a weakly positive pANCA. Blood cultures showed an enterococcal bacteremia for which the patient was treated. Skin biopsy found diffuse dermal neutrophilic infiltrate and fat necrosis without evidence of infection or vasculitis. While initially not performed, lipase and amylase were eventually tested and were elevated at 11,016 units/L and $2284 \mathrm{IU} / \mathrm{L}$ respectively. Abdominal CT imaging suggested evidence of a chronic pancreatitis while also demonstrating a multi bacterial pancreatic abscess which was drained transcutaneously and treated with appropriate antibiotics. Synovial fluid analysis from a knee aspirate demonstrated a milky yellow fluid on gross appearance. Microscopy revealed multiple fat globules, white cell count $60,000 \times 10^{6} / \mathrm{L}$, positive birefrigent crystals in an array like pattern under polarized light microscopy (see Figure 2), and negative synovial aspirate culture. Detailed lipid analysis with gas chromatography showed high levels of lipid compared to a synovial aspirate from a patient with gout used as a control, including higher levels of cholesterol and various forms of triglycerides (see Table 1).

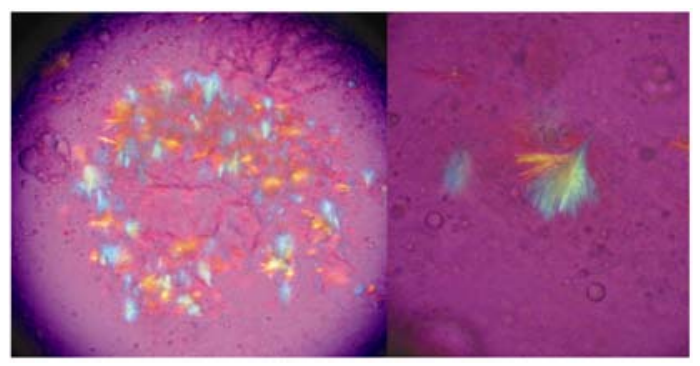

Figure 2. Microscopic appearance of patient's synovial fluid aspirate (knee) demonstrates positively birefrigent lipid crystals in an array like pattern, and fat globules. 
Table 1. Lipid Analysis of Patient's Synovial Fluid Aspirate compared to a Synovial Fluid Aspirate from a patient with gout

\begin{tabular}{lll}
\hline & Control (mcg) & Patient Sample (mcg) \\
\hline Monoglyceride (C21) & 0.84988 & 0.85586 \\
Cholesterol & 7.92485 & 16.98979 \\
Standard & 2.00000 & 2.00000 \\
Phospholipid 1 (C35) & 5.49491 & 9.61668 \\
Phospholipid 2(C37) & 4.47764 & 5.46946 \\
Phospholipid 3(C39) & 0.41939 & 5.36995 \\
Phospholipid 4(C41) & 0.36421 & - \\
Phospholipid 5 & 0.84568 & 1.04573 \\
Cholesterol Ester (C43) & 1.73716 & 1.12839 \\
Cholesterol Ester (C45) & 6.24350 & 3.62159 \\
Triglyceride (C51) & 0.08362 & 0.21074 \\
Triglyceride (C53) & 0.90330 & 2.33677 \\
Triglyceride (C55) & 2.45605 & 7.45284 \\
Triglyceride (C57) & 2.89997 & 15.14353 \\
Total & 36.70015 & 71.24132 \\
\hline
\end{tabular}

Despite normalization of the pancreatic markers and treatment with both oral and intra-articular glucocorticoids, his joint pain and swelling worsened. Serial X-rays from the time of admission and 6 weeks later demonstrated the development of multiple osteolytic bone lesions about the involved joints (see Figure 3). Pamidronate was infused monthly as a novel treatment option in an attempt to prevent further bone destruction, but was deemed unhelpful and was discontinued. Symptomatic management for pain control proceeded. The patient succumbed to complications of his immobility within a year of diagnosis.

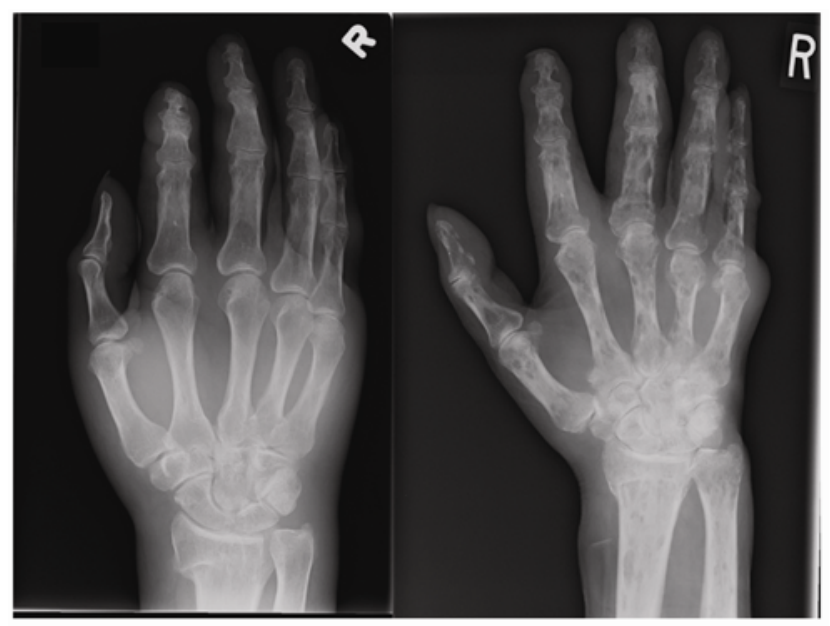

Figure 3. X-ray of the patient's right hand on day 0 (left) and six weeks later (right). The latter film demonstrates extensive and innumerable bilateral symmetric well defined predominantly intra-medullary lytic lesions involving all digits and the distal radius and ulna, resulting in endosteal scalloping, cortical thinning and frank cortical breach in numerous locations. 


\section{Discussion}

The syndrome of lobular panniculitis, pancreatitis and polyarthritis is rarely reported, with less than 30 cases found in the literature over the past 30 years ${ }^{[1-6]}$. The exact etiology remains unknown; it is hypothesized the joint manifestations of PPP syndrome are induced by a massive release of pancreatic enzymes which bind periarticular adipose tissue, leading to lypolysis of triglycerides to free fatty acids; they in turn induce a local inflammatory response.

Our patient is typical of previously reported cases, a middle aged Caucasian male with a history of alcohol abuse and a previously unidentified asymptomatic chronic pancreatitis ${ }^{[1]}$. An associated abscess or pseudocyst is also common. PPP syndrome is compared to other similar diseases, such as Weber-Christian disease, which is more common in younger females. The panniculitis also differs, with PPP syndrome uniquely demonstrating areas of fat necrosis which contain ghost-like fat cells. Joint involvement includes the small joints of the hands and feet, but also larger joints such as the knees and ankles. Bursa involvement has also been reported ${ }^{[7]}$.

An initial diagnosis of PPP syndrome is often delayed. Similar to our case, pancreatitis is often not considered to be present as a majority of cases lack abdominal symptoms. However, identification of pancreatitis is crucial as early treatment of the underlying pancreatic disease may yield the best outcome for patients.

There are a number of articular clues to identify a case of PPP syndrome which is ably demonstrated in our case. Synovial fluid analysis can rule out alternate possibilities, including rheumatoid arthritis or gout. Positive birefrigent lipid crystals, as seen in our case but not with the typical Maltese cross pattern commonly associated with lipid crystals, can be observed, but is not present in all cases. At least one report has demonstrated crystals with negative birefringence ${ }^{[8]}$. Further, lipid analysis of the aspirate is informative by demonstrating high lipid content for cholesterol and triglycerides. This case may be the first to demonstrate the aspirate findings in such detail. The radiologic findings are also profound, with plain $\mathrm{x}$-rays showing osteolytic lesions and moth-like destruction about affected joints. This corresponds to lipid infiltration, which has been confirmed by MRI in previously reported cases ${ }^{[9]}$.

Treatment options directed at the arthritis itself have demonstrated discouraging results, with corticosteroids and NSAIDs providing only symptomatic relief to some patients, but not disease regression. Functional sequelae and/or death is often reported similar to this case.

In conclusion, PPP syndrome is a rare cause of arthritis. Early diagnosis and treatment of the underlying pancreatic disease remains imperative for possible recovery. When considering this syndrome in a patient, ordering pancreatic enzymes is a key factor to achieve this goal, while we have also demonstrated the potential value of a lipid analysis of synovial aspiration too.

\section{Acknowledgements}

Thank you to Carole Johnston \& Audric Moses who both contributed to this case with their help in the analysis of the synovial fluid.

\section{References}

[1] Narvaez J, Bianchi MM, Santo P, de la Fuente D, Rios-Rodriguez V, Bolao F, et al. Pancreatitis, panniculitis, and polyarthritis. Semin Arthritis Rheum. 2010 Apr; 39(5): 417-423. http://dx.doi.org/10.1016/j.semarthrit.2008.10.001

[2] Borowicz J, Morrison M, Hogan D, Miller R. Subcutaneous fat necrosis/panniculitis and polyarthritis associated with acinar cell carcinoma of the pancreas: a rare presentation of pancreatitis, panniculitis and polyarthritis syndrome. J Drugs Dermatol. 2010 Sep; 9(9): 1145-1150.

[3] Mustafa KN, Hadidy A, Shoumaf M, Razzuki SA. Polyarthritis with chondronecrosis associated with osteonecrosis, panniculitis and pancreatitis. Rheumatol Int. 2010 Jul; 30(9): 1239-1242. http://dx.doi.org/10.1007/s00296-009-1046-9 
[4] Harris MD, Bucobo JC, Buscaglia JM. Pancreatitis, panniculitis, polyarthritis syndrome successfully treated with EUS-guided cyst-gastrostomy. Gastrointest Endosc. 2010 Aug; 72(2): 456-458. http://dx.doi.org/10.1016/j.gie.2009.11.040

[5] Jose T, Biju IK, Kumar A, Anver PC, Kuruvila R, Kuruvila S, et al. 'Pancreatitis, polyarthritis, panniculitis syndrome' (PPP syndrome) plus prolonged pyrexia-a rare presentation of chronic pancreatitis. Indian J Gastroenterol 2010 Jan 4. http://dx.doi.org/10.1007/s12664-009-0072-6

[6] Fraisse T, Boutet O, Tron AM, Prieur E. Pancreatitis, panniculitis, polyarthritis syndrome: an unusual cause of destructive polyarthritis. Joint Bone Spine. 2010 Dec; 77(6): 617-618. http://dx.doi.org/10.1016/j.jbspin.2010.05.005

[7] Halla JT, Schumacher HR,Jr, Trotter ME. Bursal fat necrosis as the presenting manifestation of pancreatic disease: light and electron microscopic studies. J Rheumatol. 1985 Apr; 12(2): 359-364.

[8] Smukler NM, Schumacher HR, Pascual E, Brown S, Ryan WE, Sadeghian MR. Synovial fat necrosis associated with ischemic pancreatic disease. Arthritis Rheum. 1979 May; 22(5): 547-553. http://dx.doi.org/10.1002/art.1780220517

[9] Price-Forbes AN, Filer A, Udeshi UL, Rai A. Progression of imaging in pancreatitis panniculitis polyarthritis (PPP) syndrome. Scand J Rheumatol. 2006 Jan-Feb; 35(1): 72-74. http://dx.doi.org/10.1080/03009740500228073 